\title{
POR UN REGRESO AL SENTIDO ORDINARIO DE LA PALABRA "INVERSIÓN"
}

\author{
Eduardo Silva Romero ${ }^{1}$
}

1. Las palabras y su empleo nunca son inocentes.

2. Las palabras jurídicas y su empleo por abogados y árbitros internacionales no son la excepción. El naciente (y todavía confuso) derecho internacional de las inversiones, si en realidad existe, ilustra, quizás más que otras ramas del derecho tradicionales (en donde -como en el derecho civil de las obligaciones y los contratos- el paso de los años -traducido en precedentes e historia- ya ha hecho su trabajo y aclarado muchas confusiones), la importancia de las palabras y su empleo en el derecho. Las (increíblemente) inagotables controversias alrededor, por ejemplo, de las nociones de "inversionista", "inversión", "trato justo y equitativo de una inversión" y "expropiación indirecta" constituyen prueba irrefutable de lo anterior².

1. Socio del equipo de arbitraje internacionat de Dechert LLP. Ha actuado como abogado y árbitro en arbitrajes intemacionales ad-hoc (CNUDMI) y administrados por el CIADI, la CCI, la LCIA, la Cámara de Comercio de Estocolmo, la Cámara de Comercio de Bogotá y otras institutciones arbitrales. Es profesor de ceoría general del derecho y contratos internacionales en el Instituto de Estudios Políticos de Paris (Sciences Po), de arbitraje en la Universidad de Paris-Dauphine (Parts IX) y de derecho internacional en la Universidad del Rosario (Bogotá).

2. Farouk Yala, "The Notion of "Investment" in ICSID Case Law: A Drifting Jurisdictional Requirement? Some "Un-Conventional" Thoughts on Salini, SGS and Mibaly", Sournal of International Arbitration, Vol. 22 No. 2 (2005), pp. 105 - 125; Barton Legum, "Defining Investment and Investor: Who is Entitled to Claim?", Arbitration International, Vol, 22 No. 4 (2006), pp. 521 526; Walid Ben Hamida, "Two Nebulous ICSID Features: The Notion of Investment and the Scope of Annulment Control", Journal of International Arbitration, Vol, 24 No. 3 (2007), pp. 287 - 306; Yulia Andreeva, "The Tribunal in Malaysian Historical Salvors v. Malaysia Adopts a Restrictive Interpretation of the Term "Investment", , Journal of International Arbitration, Vol. $25 \mathrm{No} .4$ (2008), pp. 503 - 506; Anna Turinov, "Investment" and "Investor" in Energy Charter Treaty Arbitration: Uncertain Jurisdiction", Joumal of International Arbitration, Vol. 26 No. 1 (2009), pp. 1 - 23; Stephen Fietta, "Expropriation and the "Fair and Equitable" Standard. The Developing Role of 
3. Nos concentraxemos, en estas páginas dedicadas a la nueva Revista Ecuatoriana de Arbitraje, a pensar en voz alta y borrador (razón por la cual las observaciones que siguen no deben ser tomadas en serio) sobre las controversias (más o menos recientes) alrededor de la noción de "inversión".

4. No sobra recordar que la definición de la noción de "inversión" es crucial, antes que nada, para determinar y circunscribir la jurisdicción arbitral resultante de tratados bilaterales de promoción y protección de inversiones ("TBI" en singular y "TBIs" en plural) y del Convenio sobre Arreglo de Diferencias Relativas a Inversiones entre Estados y Nacionales de Otros Estados suscrito en Washington en 1965 (el "Convenio de Washington").

5. Olvidemos, por unos breves instantes, el lenguaje jurídico y el prisma a través del cual éste nos hace percibir la realidad. ¿Qué sentido podríamos entonces atribuirle a la palabra "inversión" desde el sentido común y su instrumento preferido, esto es, el profano lenguaje ordinario? ¿Qué quiere, por ejemplo, decir un estudiante cuando afirma que "sus estudios de especialización son una inversión para el futuro"? ¿Qué quiere decir, también por ejemplo, aquél que sostiene que "no existe mejor inversión que la que se hace en la piedra" (léase en bienes inmuebles)? ¿Y qué quiere decir el gerente de una empresa multinacional cuando asegura que "dicha empresa ha hecho una inversión gigantesca para modernizar una planta de tratamiento de aguas operada por concesión"?

Investors" "Expectations" in International Invesiment Arbitration", Journal of International Arbitration, Vol. 23 No. 5 (2006), pp. 375 - 399; Lucy Reed y Daina Bray, "Fair and Equitable Treatment: fairly and equitably applied in Lieu of Unlawful Indirect Expropriation?", In: Contemporary Issues in International Arbitration and Mediation: the Fordham Papers 2007, Nijhoff (2008); J. Roman Picherak, "The Expanding Scope of the Fair and Equitable Treatment Standard: Have Recent Tribunals Gone Too Far?". The Journal of World Investment \& Trade, Vol. 9, No. 4, 2008 pp. 255-291; Jean Kalicki y Suzanna Medeiros, "Fair, Equitable and Ambigeous : What is Fair and Equitable Treatment in International Investment Law?", JCSID Review, vol. 22, No. 1, 2007, pp. 24-54. 
6. El fundador de la filosofía del lenguaje, Ludwig Wittgenstein 3', quien, sin lugar a dudas, habría utilizado muchos más ejemplos antes de formular preguntas más abstractas o sugerir conclusiones, se hubiera preguntado, después de describirlos, si los tres personajes de más arriba se refirieron, en sus dichos, a una misma noción o idea de "inversión". Quizás no. Entre los dichos de los tres personajes, sin embargo, parecen existir "aires de familia" o trazos comunes que, de alguna manera, muestran rasgos característicos de lo que alguien podría aventurarse a llamar "inversión". Para los tres personajes, en efecto, la palabra "inversión" parece evocar la conjunción entre un esfuerzo en tiempo y / o dinero y la obtención de un rédito al final de un período más o menos indeterminado.

7. El "esfuerzo", el paso de cierto tiempo -la "duración"- y la "probable obtención de un beneficio" parecen ser elementos esenciales de la noción de "inversión" en el lenguaje ordinario. Todos, después de todo, parecemos emplear la palabra "inversión" y el verbo "invertir", en el lenguaje de todos los días, percibiendo en ella los inherentes "esfuerzo", "duración" y "probable obtención de un beneficio".

8. El ejemplo de la inversión gigantesca en la planta de tratamiento de aguas sugeriría, además, que la inversión, cuando el "esfuerzo" lo hace una empresa extranjera en un Estado, debe contribuir al desarrollo económico de este último.

9. El derecho, sin embargo, parece detener el extraordinario poder de modificar (tenemos la tentación de decir "dero-

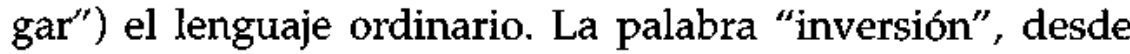
una 6ptica jurídica, parece ser empleada con alguna regularidad de una forma completamente distinta de aquélla en que se usa en el lenguaje ordinario, esto es, de una manera

3. Eduardo Silva Romero, Wittgenstein et la philosophie du droit, PUF (2002). 
ajena a los criterios del "esfuerzo", la "duración" y la "probable obtención de un beneficio".

10. Los TBIs, en especial, comprenden un artículo (por lo general el primero) en el que, según se afirma generalmente, se "define", a través de una lista enumerativa (no taxativa), el término de "inversión"4 $\sin$, al menos a primera vista, tomar en cuenta los criterios mencionados.

11. Varios tribunales arbitrales, por lo general obrando bajo el imperio del Reglamento de Arbitraje de la Comisión de las Naciones Unidas para el Derecho Mercantil Internacional ("CNUDMI"; "UNCITRAL", en inglés), han sostenido en los últimos años, frente a Estados soberanos que han objetado a su competencia arguyendo que la operación económica del caso no constituía una "inversión", que la palabra "inversión" debe ser definida exclusivamente sobre la base de la lista de "inversiones" contenida en el TBI aplicable. Este método de definición de la noción de "inversión" ha sido calificado de "subjetivo" 5 . Su aplicación, en nuestra opinión, conduciría a un "nominalismo" ${ }^{6}$ insoportable (1).

12. Otros tribunales arbitrales, obrando -en su gran mayoríade conformidad con el Convenio de Washington, han preferido, quizás guiados, antes que nada, por el sentido común, corregir dicha lista de "inversiones" (demasiado amplia) comprendida en los TBIs y definir la noción de "inversión"

4. Rudolf Dolzer y Margrete Stevens, Bilateral investment treaties, Nijhoff (1995); Jose Luis Siqueiros, "Bilateral treaties on the reciprocal protection of foreign investment", California Western International law Journal, vol. 24, No. 2, 1994, pp. 255-275; Andrew Newcombe, Law and practice of investment treaties: standards of treatment, Kluwer (2009); Giorgio Sacerdoti, Bilateral treaties and multilateral instruments on investment protection, Rectueil des cours, Vol. 269, 1997, pp. 251460 .

5. Malaysian Historical Salvors, SDN, BHD c./Malasia, Caso CIADI No. ARB/05/10, Opinión disidente del Juez Shahabuddeen a la decisión de anulación, par. 7 , en línea : < bttp://ta.law.uvic.ca/documents/MalaysianHistoricalAnnulmentDissent.pdf $>$ (en adelante, "Opinión Shahabuddeen").

6. La posición nominalista sostiene que las especies y géneros no son realidades anteriores a las cosas -"esencias", capaces de ser conocidas por abstracción-, sino que se trata, simplemente, de nomina (nombres) o voces, que utilizamos para designar a grupos de individuos. "Nominalismo", in: Enciclopedia de la Cultura Española, Tomo 4, pp. 519-520, Editora Nacional (1967). 
identificando -sobre la base de fuentes externas al TBI aplicable- criterios determinantes más o menos objetivos de lo que sería una "inversión". Este método de definición de la noción de "inversión" ha sido calificado de "objetivo"7. Su aplicación, en nuestra opinión, conduciría a un "convencionalismo realista" 8 más o menos convincente (2).

\section{Del Nominalismo en la Definición de "INVERsión"}

13. El artículo 1(1) del TBI celebrado entre la República del Ecuador y Costa Rica, por ejemplo, dispone lo que sigue:

(1) El término "inversión" designa de conformidad con las leyes y reglamentos de la Parte Contratante en cuyo territorio se realizó la inversión, todo tipo de activo invertido por inversionistas de una Parte Contratante en el territorio de la otra Parte Contratante. Incluye en particular, aunque no exclusivamente:

(a) La propiedad de bienes muebles e inmuebles, así como los demás derechos reales tales como hipoteca, derechos de prenda, usufructos y derechos similares;

(b) Acciones, títulos, obligaciones, cuotas societarias, y cualquier otro tipo de participación en sociedades;

(c) Derechos y obligaciones o créditos directamente vinculados a una inversión específica;

(d) derechos de propiedad intelectual, incluyendo, en especial, derechos de autor y derechos conexos y derechos de propiedad industrial, tales como: marcas de fábrica o de comercio, indicaciones geográficas, dibujos, patentes, diseños industriales, esquemas de trazado (topografías) de los circuitos integrados, know-how, derecho de obtentores de variedades vegetales y otros derechos similares;

7. Opinión Shahabuddeen, par. 8.

8. Las reglas de derecho, como las reglas de gramática, son convencionales en el sentido que pueden ser creadas arbitrariamente por el hombre. Sin embargo, el carácter arbitratio de las reglas de derecho, como de las reglas de gramática, está limitado por la realidad y, en particular, por la realidad humana. El derecho, y el lenguaje juridico, es entonces una convención humana moderada por las indicaciones de la realidad y de las formas de vida (valores). Eduardo Silva Romero, Wittgenstein et la philosophie du droit, PUF (2002), p. 337. 
(e) Concesiones económicas conferidas por ley y por contrato incluidas las concesiones para la prospección, cultivo, extracción o explotación de los recursos naturales; y

(f) La reinversión de utilidades.

14. Basta con revisar con cuidado la lista de "inversiones" de más arriba para percatarse de que lo allí listado son, más que "inversiones" según el sentido común, "activos".

15. El título del $\mathrm{TBI}$, sin embargo, se refiere a la promoción y protección de "inversiones" y no a la "promoción" y protección de "activos". La palabra "inversión", en nuestra opinión, no pudo haber sido utilizada en los TBIs como sinónimo de "activo" puesto que, entre otras razones, la frase "promoción de activos" es un sin sentido. Si bien es cierto, en efecto, que tanto "inversiones" como "activos" pueden ser "protegidos", solamente aquéllas pueden ser -sin que se abuse del lenguaje- "promovidas".

16. La considerable violencia que dichas listas de "inversiones" cometen contra el sentido ordinario de la palabra "inversión" nos conduce, por lo tanto, a preguntarnos si lo que allí se lista representa, más que "inversiones", formas jurídicas y económicas que puede revestir una "inversión". La manera (desafortunada) en que los encabezados de dichas listas han sido redactados, sin embargo, parece más bien indicar que los ítems listados son realmente "inversiones".

17. A esta última conclusión han llegado recientemente tres tribunales arbitrales ad-hoc obrando según el Reglamento de Arbitraje de la CNUDMI.

18. En el caso Swembalt c./ Lituania ${ }^{9}$, en primer lugar, el tribunal arbitral llegó a la conclusión de que la compraventa de un navío destinado a un centro de negocios flotante era, según

9. Swembalt AB, Sweden c/ Lituania, Laudo (CNUDMI) del 23 de octubre de 2000, par. 26-35, en línea: <http:/ita.law.uvic.ca/documents/Swembalt-Latvia-Award-230ct2000.pdf> 
el TBI aplicable, una "inversión", desestimó las objeciones a la competencia presentadas por Lituania y decidió, en un laudo final, condenar a Lituania a pagar una suma de dinero. Lituania no cumplió con dicho laudo voluntariamente. Swembalt, empresa sueca, por consiguiente, intentó entonces ejecutar el laudo arbitral de manera forzosa en Dinamarca ante la Corte Marítima y Comercial de Copenhague. Lituania, por su lado, se opuso a la ejecución de dicho laudo arguyendo que un navío comprado con destino a un centro de negocios flotante no constituía una "inversión" de conformidad con el TBI entre Suecia y Lituania. La Corte Marítima y Comercial de Copenhague, sin embargo, confirmó la definición de "inversión" adoptada por el tribunal arbitral y agregó que la palabra "inversión" comprendida en el TBI debía ser interpretada de una manera amplia y no restringida ${ }^{10}$.

19. ¿La compraventa de un navío destinado a un centro de negocios flotante es una "inversión"? ¿Una compraventa, en términos generales, supone una "duración" y "la probable obtención de un beneficio" al final de un período más o menos indeterminado?

20. Un segundo tribunal arbitral, en el caso Mytilineos Holdings $S A$ c./ La Unión de Serbia \& Montenegro y la República de Serbia ${ }^{11}$, también decidió desafiar el sentido común y llegó a la conclusión, ante objeciones a su competencia esgrimidas por las demandadas, de que un contrato de sociedad (partnership) por medio del cual se le entregaban capital y repuestos a una empresa estatal minera y se asumía la obligación de adquirir parte de su producción constituía una inversión según los términos del TBI aplicable.

10. República de Lituania c./ SwemBalt Aktiebolag, decisión de la Corte Marítima y Comercial de Copenhague (2003), Caso S-22-01, in: Sigvard Jarvin y Annette Magnusson, International Arbitration Court Decisions, Jure AB (2008).

11. Mytilineos Holdings SA c./ La Unión de Serbia \& Montenegro y la República de Serbia, laudo patcial (CNUDMI) sobre la competencia del 8 de septiembre de 2006, par. 114 y 115 (en adelante "Mytilineos"), en línea: < http://ita.law.uvic.ca/documents/MytilineosPartial/Award.pdf >. 
21. Las demandadas, sin embargo, invocaban los criterios determinantes de una "inversión" desarrollados por la jurisprudencia arbitral del Centro Internacional de Arreglo de Diferencias Relativas a Inversiones (el "CIADI") para demostrar que la operación económica descrita más arriba no era una "inversion", y el tribunal arbitral, por lo tanto, no era competente para conocer del fondo del asunto.

22. El tribunal arbitral en Mytilineos Holdings SA c./ La Unión de Serbia \& Montenegro y la República de Serbia, sin embargo, descartó, a través de un manifiesto metodológico, la aplicación de la jurisprudencia CIADI de la siguiente manera:

"It is the established practice of ICSID tribunals to assess whether a specific transaction qualifies as an "investment" under the ICSID Convention, independently of the definition of investment in a BIT or other applicable investment instrument, in order to fulfill the ratione materiae prerequisite of Article 25 of the Convention. This requirement is set out in Article 25(1) of the Convention which confines the jurisdiction of ICSID arbitration tribunals to "legal dispute[s] arising directly out of an investment" without defining "investment."

[...]

However, this latter ratione materiae test for the existence of an investment in the sense of Article of the 25 ICSID Convention is one specific to the ICSID Convention and does not apply in the context of ad hoc arbitration provided for in BITs as an alternative to ICSID.

In the present ad hoc arbitration under the UNCITRAL Rules one would therefore have to conclude that the only requirements that have to be fulfilled in order to confer ratione materiae jurisdiction on this Tribunal are those under the BIT"12.

23. ¿Reúne dicho contrato de partnership las características de la "inversión" según el sentido común y el lenguaje ordinario?

12. Mytilineos, par. 114, 117-118.

224 Revista ECuAtorlana de ARbitraje 
24. Otro tribunal arbitral CNUDMI, por último, en el caso Pren Nreka c./ la República Checa ${ }^{13}$, decidió, también ante objeciones a su competencia, que un contrato de renovación y arrendamiento de inmueble con el fin de instalar una pizzería celebrado entre un particular y la Administración era una "inversión" según los términos del TBI entre Croacia y la República Checa.

25. Dado que la sede de dicho arbitraje era París, la República Checa interpuso un recurso de anulación contra dicho laudo arbitral ante la Corte de Apelaciones de París.

26. La Corte de Apelaciones de París ${ }^{14}$ rechazó el recurso y confirmó la definición adoptada por el tribunal arbitral afirmando lo que sigue:

Considerando que las disposiciones del TBI transcritas no prevén un criterio para caracterizar lo que constituye una inversión sino que dan tan sólo una enumeración, que además no es limitativa, de los casos en los cuales se considera que hay una inversión, que las condiciones enunciadas por la República Checa sobre la definición de la inversión añaden al texto del TBI el cual no prevé que sea una condición de la competencia;

Que el único test que se debe realizar es el de determinar si la operación objeto de la demanda entra o no en el marco de las disposiciones del TBI para verificar si puede efectivamente beneficiarse de la oferta de arbitraje del tratado, esta exigencia siendo

13. Pren Nreka c//la República Checa, laudo (CNUDMI) de febrero de 2007, no publicado. La decisión de la Corte de Apelaciones de París (Primera Cámara - Sección C) del 25 de septiembre 2008 resume, sin embargo, dicho laudo, en linea: 〈http:/ita.law.uvic.ca/documents/Nrekaarret.pdf $>$.

14. Traducción del autor: "Considérant que les dispositions du TBI qui viennent d'être rappelées ne fournissent pas de critère pour caractériser ce qu' est un investissement mais donnent seulement une énumération, et encore de manière non limitative, des cas considérés comme des investissements, que les conditions soulevées par la République tchèque quant à la définition de l'investissement ajoutent au texte du TBI lequel n'en fait pas une question de compétence :Que le seut test est celut de savoir si l'opération qui sert de base à la demande entre dans le cadre des prévision du TBI pour vérifier si elle peut effectivement bénéficier de l'offre d'arbitrage du traité, cette exigence étant en l'espèce réalisée pour des locaza cédés à bail pendant IS ans en contrepartie de l'exécution de travara de rénovation qui constituent un investissement selon les termes très larges du TBI que l'on a rappelés et quí visent notamment $n$ 'importe quel droit contractuel'. Decisión de la Corte de Apelaciones de París (Primera Cámara - Sección C) del 25 de septiembre 2008, p. 5, en linea: <http:/ita.law.uvic.ca/documents/Nrekaarret.pdfs. 
satisfecha en lo que concierne los locales cedidos en arrendamiento por 15 años en contraprestación de la realización de trabajos de renovación que constituyen una inversión según los términos muy amplios del TBI que hemos recordado y que incluyen en particular cualquier derecho contractual.

27. ¿La famosa pizzería constituye realmente una "inversión"?

28. En los tres casos arriba citados, los Estados demandados buscaban, argumentando, en especial, que las operaciones económicas en causa no "contribuían al desarrollo económico de los Estados receptores de la supuesta "inversión"”, que tribunales arbitrales y cortes restringieran la definición de "inversión" que se puede deducir de las listas de "inversiones" -que se traduce en decir que "todo es inversión" o que "inversión" y "activos" son sinónimos- a través de criterios más o menos extrínsecos al TBI aplicable. Los Estados demandados, en suma, defendían la existencia de una noción única de inversión-cuyos elementos constitutivos comprendían, en particular, "la contribución al desarrollo económico del Estado receptor de la inversión"- que habría sido descrita, en especial, por la jurisprudencia CIADI.

29. Tribunales arbitrales y cortes, sin embargo, respondieron, explícita o implícitamente, que criterios extrínsecos al TBI $-\mathrm{y}$, en especial, criterios precisados por tribunales arbitrales CIADI- no podían ser aplicados para corregir la noción de "inversión" que se deduce de la lista de "inversiones" comprendida en los TBIs. El método subjetivista, en este sentido, consistiría en que "the jurisdiction of [the Tribunal] meant to be solely dependent on the will of the parties"15.

30. Un tribunal arbitral CNUDMI responsable de la resolución de una controversia en materia de "inversión", en definitiva, no debería, según dichos tribunales y cortes, aplicar la jurisprudencia CIADI para determinar, entre otras cosas, la

15. Opinión de Shahabuddeen, par. 62. 
noción de "inversión" puesto que las disposiciones del Convenio de Washington no le son aplicables.

31. Parece, sin embargo, totalmente ilógico que -como parece sostenerlo el "subjetivismo"-- puedan existir tantas nociones de "inversión" como TBIs han sido concluidos en el mundo.

32. Es aún más absurdo que se sostenga, explícita o implícitamente, que la noción de "inversión" depende del mecanismo de resolución de litigios previsto en el TBI escogido por el inversionista. No parece, en efecto, lógico que una misma operación económica realizada, por ejemplo, en territorio ecuatoriano pueda ser calificada de "inversión" por un tribunal CNUDMI y no lo sea por un tribunal arbitral CIADI.

33. Que la definición de "inversión" dependa del mecanismo de resolución de litigios previsto en el TBI escogido por el inversionista supone, además, que, cuando un inversionista sospeche que un tribunal arbitral CIADI (por lo general más "objetivista") pueda considerar que la operación económica en cuestión no es una "inversión", dicho inversionista prefiera comenzar un arbitraje CNUDMI contra el Estado con la esperanza de que, gracias a la aplicación del método "subjetivo", el tribunal arbitral CNUDMI se limite a determinar si la operación económica del caso corresponde con alguno de los ítems de la lista de "inversiones" del TBI.

34. ¿La estrategia anterior no implica un abuso del derecho, esto es, un abuso del proceso de resolución de desavenencias previsto en los TBIs? ¿Dicha estrategia no sería contraria aI sacrosanto principio de la buena fe?

35. Es quizás por lo anterior que varios tribunales, especialmente obrando bajo los auspicios del CIADI, han preferido adoptar y desarrollar -siguiendo en esto una filosofía del lenguaje "convencionalista-realista" - una noción única de "inversión". 


\section{Del Convencionalismo Realista en la Noción DE "INVERSIÓN"}

36. Detrás de la defensa de una noción única y "objetiva"16 de "inversion" se oculta, sin lugar a dudas, la garantía del valor -fundamental en todas latitudes- de la seguridad jurídica.

37. Es evidentemente contrario a la seguridad jurídica que la palabra "inversión" pueda revestir múltiples sentidos y que los inversionistas puedan escoger entre ellos para asegurar -con gran facilidad- la protección de sus "activos".

38. Varios tribunales arbitrales CIADI, dotados de sentido común y conscientes de la importancia del valor de la seguridad jurídica, han limitado la noción de "inversión" que resultaría de las listas de "inversiones" comprendidas en los TBIs a través de tres métodos distintos (2.1, 2.2 y 2.3).

\subsection{De la limitación de la lista de "inversiones" de los TBIs a través deI Convenio de Washington}

39. Fueron en especial los tribunales en los casos Salini ${ }^{17}$ y Fedax ${ }^{18}$ los que, a través del Convenio de Washington, decidieron limitar la amplísima noción de "inversión", contraria al sentido común y el lenguaje ordinario que lo expresa, que resultaría de las listas de "inversiones" de los TBIs.

40. En el caso Salini, en primer lugar, el tribunal arbitral, recurriendo al Convenio de Washington, definió cuatro elemen-

16. Por el contrario, el objetivismo, según el juez Shahabuddeen, significa quc la competeneia de un tribunal CIADI "(is) meant to be dependent on the will of the parties subject to conformity with the overriding objectives of ICSID as a body concerned with the economic development of the host State", Opiniơn de Shahabuddeen, par. 62.

17. Salini Construtorri S.p.A. e Italstrade S.p.A. c./Marruecos, Caso CIADI No. ARB/00/4, decisión sobre la jurisdicción del 23 de julio de 2001, Journal de Droit International, 2002, p. 196 (en adelante "Salini").

18. Fedax N.Y. c./Venezkela, Caso CLADI No. ARB/96/3, decisión sobre la jurisdicción del 11 de julio de 1997, par. 22-32, en línea: <bttp://ita.law.uvic.ca/documents/Fedax-1997-Last.pdf?. 
tos constitutivos de la noción de inversión de la siguiente manera:

"La doctrina considera generalmente que una inversión supone aportes, cierta duración de ejecución del mercado y una participación en los riesgos de la operación. A la lectura del preámbulo de la Convención debe añadirse el criterio de la contribución al desarrollo económico del Estado huésped de la inversión" ${ }^{\prime 19}$.

41. Dado que el Convenio de Washington no comprende una definición (al menos explícita) de "inversión", el método utilizado por el tribunal arbitral Salini para limitar la noción de "inversión" del TBI entre Italia y Marruecos no deja de causar, desde un punto de vista jurídico, sorpresa. ¿De qué fuente, en efecto, provienen los cuatro elementos constitutivos de la noción de "inversión" identificados por el tribunal arbitral Salini? ¿Dichos elementos provienen realmente del Convenio de Washington?

42. En realidad de verdad, dichos elementos parecen, más bien, provenir del sentido común y su lenguaje ordinario. No podemos resistir la tentación de subrayar que al menos los tres primeros elementos identificados por el tribunal Salini nos parecen formar evidentemente parte de la noción de "inversión". Dichos elementos, en efecto, coinciden con los trazos comunes del "esfuerzo", la "duración" y "la probable obtención de un beneficio" -este último elemento recogería el elemento del "riesgo" - mencionados más arriba.

43. El método utilizado en el caso Salini, quizás porque el sentido común es, como escribiera Descartes ${ }^{20}$, una de las cosas

19. Traducción del autor: "La doctrine considère généralement que l'investissement suppose des apports, une certaine durée d'exécution du marché et une participation atx risques de l'opération. La lecitre du Préambule de la Convention permet d y ajouter le critère de la contribution au développement économique de l'Etat d'accueil de l'investissement", Salini, par. 53.

20. "El buen sentido es lo que mejor repartido está entre todo el mundo, pues cada cual piensa que posee tan buena provisión de él, que aun los más descontentadizos respecto a cualquier otra cosa, no suelen apetecer más del que ya tienen. En lo cual no es verosimil que todos se engañen, sino que más bien esto demuestra que la facullad de juzgar y distinguir lo verdadero de lo falso, que es propiamen- 
del mundo mejor compartida, se ha vuelto famoso. Otros tribunales no han dudado en erigir dicho método en "test" $\mathrm{y}$ aplicarlo en múltiples casos ${ }^{21}$. El caso Jan de Nul, quizás, constituye el ejemplo más ilustrativo.

44. El tribunal arbitral Jan de Nul, en segundo lugar, confirmó el método Salini de la siguiente manera:

"The ICSID Convention contains no definition of the term "investment". The Tribunal concurs with ICSID precedents which, subject to minor variations, have relied on the so-called "Salini test". Such test identifies the following elements as indicative of an "investment" for purposes of the ICSID Convention: (i) a contribution, (ii) a certain duration over which the project is implemented, (iii) a sharing of operational risks, and (iv) a contribution to the host State's development, being understood that these elements may be closely interrelated, should be examined in their totality and will normally depend on the circumstances of each case $^{\prime \prime 22}$.

45. Parecería, sin embargo, que (solamente) uno de los elementos identificados por el tribunal Salini -el cuarto- ha dado lugar a controversia y debate. Emmanuel Gaillard23, por ejemplo, sostiene que "la contribución al desarrollo económico del Estado receptor de la inversión" no es un elemento de la noción de "inversión" o, a lo sumo, se halla com-

te lo que llamamos buen sentido o razón, es naturalmente igual en todos los hombres; $y$, por lo tanto, que la diversidad de nuestras opiniones no proviene de que unos sean más razonables que otros, sino tan sólo de que dirigimos nuestros pensamientos por derroteros diferentes y no consideramos las mismas cosas", René Descartes, Discurso del método, Primera Parte, Edaf S.A. (2001), p. 2.

21. Por ejemplo: Saipem S.p.A. c./Bangladesh, Caso CIADI No. ARB/05/07, decisión sobre la competencia del 21 de marzo de 2007, par. 99, en línea: <http://ita.law,uvic.ca/documents/SaipemBangladest-Jurisdiction.pdfs; Phoenix Action. Lid. c/República Checa, Caso CIADI No. ARB/06/5, laudo del 15 de abril de 2009, par. 81, en línca: 4 http//ita.law.uvic.ca/documents/PhoenixAward.pds (en adelante "Phoenix"); Bayindir Insaat Turizm Ticaret Ve Sanayi A.S. c./ Pakistán, Caso CIADI No. ARB $/ 03 / 29$, decisión sobre la competencia del 14 de noviembre de 2005, par. 130, en línea: $<$ http:/ita.law.uvic.ca/documents/Bayindr-jurisdiction.pds $\$$.

22. Jan de Nul N.V. and Dredging International N.V. c./ Egipto, Caso CIADI No. ARB/04/13, decisión sobre la competencia del 16 de junio de 2006 , par. 91, en linea: $<\mathrm{http} / /$ ita.law.uvic.ca/documents/JandeNuljurisdiction06 $1606 . p d \mathrm{~d}$.

23. E. Gaillard, "Chronique des sentences arbitrales", Journal de droit international, 2008, p. 313. 
prendida implícitamente en los tres primeros elementos identificados por el tribunal arbitral Salini. ¿Qué tan intensa, se preguntan Gaillard y otros, debe ser "la contribución al desarrollo económico del Estado receptor de la inversión"?

46. Controversia y debate alrededor del cuarto elemento de la "inversión" según Salini dieron recientemente lugar a la decisión de un Comité CIADI ad-hoc de anulación de anular un laudo arbitral en el que un tribunal concluyó que una operación económica que no contribuye intensamente al desarrollo económico del Estado receptor de la inversión no puede ser calificada de inversión. Para el Comité CIADI adhoc del caso Malaysian Historical Salvors SDN BHD c./ el Gobierno de Malasia ${ }^{24}$, en efecto, "la contribución al desarrollo económico del Estado receptor de la inversión" no es un elemento de la noción de "inversión".

47. La decisión del Comité CIADI ad-hoc del caso Malaysian Historical Salvors SDN BHD c./ el Gobierno de Malasia puede ser criticable. Si bien es cierto que la finalidad de los TBIs es, antes que nada, la promoción y la protección de "inversiones" y que, desde cierta óptica, dichos tratados deben ser interpretados a favor de los inversionistas, también es verdad que, si los Estados aceptaron proteger la inversión extranjera a través de los TBIs, fue bajo la clara expectativa de atraerla y así impulsar el desarrollo económico de sus pueblos y territorios.

48. La decisión del Comité CIADI ad-hoc del caso Malaysian Historical Salvors SDN BHD c./ el Gobierno de Malasia de todas formas debería, en nuestra opinión, ser aprobada en la medida en que, en nuestra opinión, no es absolutamente claro que "la contribución al desarrollo económico del

24. Malaysian Historical Salvors SDN BHD c./ el Gobiemo de Malasia, Caso CIADI No ARB/05/10, decisión de anulación del 16 de abril de 2009 , par. 56 a 82, en linea: « http:/ita.law.uvic.ca/documents/MalaysianHistoricalAnnulment.pdf $\%$. 
Estado receptor de la inversión" constituya un elemento esencial de la noción de "inversión".

49. No deja, sin embargo, de ser sorprendente que el Comité CIADI ad-hoc del caso Malaysian Historical Salvors SDN BHD c./ el Gobierno de Malasia haya concluido que un contrato celebrado entre una empresa privada de salvamento de buques y sus cargamentos y un Estado para el salvamento de un cargamento de un buque zozobrado constituye, a pesar de la naturaleza eminentemente mercantil de dicho negocio jurídico, una "inversión".

\subsection{DE LA LIMITACIÓN DE LA LISTA DE "INVERSIONES" DE LOS TBIS A TRAVÉS DE LA NATURALEZa MERCANTIL DE LA OPERACIÓN ECONÓMICA EN CAUSA}

50. Los límites entre las nociones de "inversión" y "operación mercantil" no parecen ser totalmente claros en derecho internacional.

51. La Ley Modelo de la CNUDMI sobre el arbitraje comercial internacional, por ejemplo, provee, en su nota de pie de página, una amplísima lista de actividades que serían calificables de "operaciones mercantiles":

Debe darse una interpretación amplia a la expresión "comercial" para que abarque las cuestiones que se plantean en todas las relaciones de índole comercial, contractuales o no. Las relaciones de índole comercial comprenden las operaciones siguientes, sin limitarse a ellas: cualquier operación comercial de suministro o intercambio de bienes o servicios, acuerdo de distribucion, representación o mandato comercial, transferencia de créditos para su cobro ("factoring"), arrendamiento de bienes de equipo con opción de compra ("leasing"), construcción de obras, consultoría, ingeniería, concesión de licencias, inversión, financiación, banca, seguros, acuerdo o concesión de explotación, asociaciones de empresas y otras formas de cooperación industrial o comercial, 
transporte de mercancías o de pasajeros por vía aérea, marítima, férrea o por carretera.

52. De la lectura de la lista contenida en el párrafo precedente se desprendería que varias actividades que podrían ser calificadas, según el sentido común y su lenguaje ordinario, de "inversiones" serían, según la Ley Modelo de la CNUDMI, "operaciones mercantiles".

53. Será, sin embargo, evidente para aquellos juristas que conocen la distinción romano-germánica entre el derecho civil y el derecho comercial ${ }^{25}$ que el criterio de "comercialidad" adoptado en la Ley Modelo de la CNUDMI no coincide con aquél adoptado en los derechos internos de varios países. La "comercialidad" de la Ley modelo de la CNUDMI es mucho más amplia que aquélla normalmente adoptada en los derechos nacionales que establecen la distinción entre derecho civil y derecho comercial.

54. La "comercialidad" de una operación económica, a pesar de lo anterior, ha sido utilizada por un tribunal arbitral CIADI para determinar que la controversia que le fuera confiada por las partes no era una "inversión". En el caso Joy Mining Machinery Limited c./ Egipto26, en efecto, un tribunal arbitral CIADI se declaró incompetente para conocer del fondo del asunto al concluir que un contrato de compraventa, por complejo que éste sea, no puede ser una "inversión" ya que es, más bien, una operación mercantil. El análisis del tribunal arbitral en Joy Mining Machinery Limited c./ Egipto es muy interesante:

25. La distinción entre el derecho civil (derecho común) y el derecho comercial (derecho especial y derogatorio del derecho común) existe en varios paises: Alemania, Bélgica, Brasil, Colombja, España, Francia, Uruguay, etc.

26. Joy Mining Machinery Limited c./ Egipto, Caso CIADI No. ARB/03/11, laudo del 6 de agosto de 2004, en línea: <http://ita.law.uvic.ca/documents/JoyMining_Egypt.pdfs. 
"The Tribunal is also mindful that if a distinction is not drawn between ordinary sales contracts, even if complex, and an investment, the result would be that any sales or procurement contract involving a State agency would qualify as an investment. International contracts are today a central feature of international trade and have stimulated far reaching developments in the governing law, among them the United Nations Convention on Contracts for the International Sale of Goods, and significant conceptual contributions. Yet, those contracts are not investment contracts, except in exceptional circumstances, and are to be kept separate and distinct for the sake of a stable legal order. Otherwise, what difference would there be with the many State contracts that are submitted every day to international arbitration in connection with contractual performance, at such bodies as the International Chamber of Commerce and the London Court of International Arbitration?"27

55. Los travaux préparatoires del Convenio de Washington relatan cómo los arquitectos del mismo entendieron que operaciones mercantiles tales como contratos de compraventa no podían constituir "inversiones" y no entraban por ende dentro del ámbito del Convenio. Christoph $\mathrm{H}$. Schreurer ${ }^{28}$ expresa esta idea como sigue:

"[...] Transactions whose characterization as investment has been cas into doubt incluye programmes of work such as construction contracts, training programmes, technical asístanse programmes, consultants' contracts and suppliers' credits. Depending on the overall circumstances, there may or may not amount to investments. Non-recurring transactions such as simple sales and parchases of goods or short-term Commercial credits clearly do not qualify as investments".

56. Los "subjetivistas" criticarán el recurso a dichos trabajos preparatorios sobre dos bases distintas.

27. Id., par. 58 .

28. Christoph Schreurer, The ICSID Convention: A Commentory, p. 139, Cambridge (2001).

234 Revista Ecuatoriana de Arbitraje 
57. El artículo 32 de la Convención de Viena sobre el Derecho de los Tratados, por una parte, específicamente estatuye que el recurso a trabajos preparatorios es subsidiario y solamente está abierto para confirmar la interpretación del tratado a la que se llegue según el método previsto en el artículo 31 o cuando la aplicación de éste conduzca a resultados ambiguos, obscuros o irrazonables.

58. ¿No es irrazonable concluir que la venta de un navío destinado a un centro de negocios flotante es una "inversión"?

59. ¿No es también irrazonable sostener que el salvamento de un navío y su cargamento es una "inversión"?

60. Los "subjetivistas", por otra parte, pueden aducir, según lo ya dicho, que un tribunal arbitral CNUDMI no podría referirse a los trabajos preparatorios del Convenio de Washington dado que las disposiciones de éste no le son aplicables.

61. El criterio extrínseco a los TBIs de la "comercialidad", sin embargo, nos parece fundamental para moderar las listas de "inversiones" en éstos e impedir abusos del derecho. Salta, en este orden de ideas, a la vista que, de haber sido utilizado por abogados, árbitros internacionales y cortes, las operaciones económicas en causa en los casos Swembalt c./ Lituania, Mytilineos Holdings SA c./ La Unión de Serbia $\mathcal{E}$ Montenegro y la República de Serbia, Pren Nreka c./ la República Checa y Malaysian Historical Salvors SDN BHD c./ el Gobierno de Malasia nunca habrían podido ser calificadas de "inversiones" al ser esencialmente operaciones mercantiles.

62. El lector notará, sin embargo, que hemos caído en la trampa que denunciábamos al principio de este texto. Hemos (quizás) caído en la trampa de derogar el lenguaje ordinario a través del lenguaje jurídico. No parece, en efecto, muy claro que la "comercialidad" de una operación económica sea un 
elemento del sentido común y el lenguaje ordinario que permita excluir ciertas actividades de la categoría de "inversión". ¿La compraventa de un inmueble para la reventa, por ejemplo-operación mercantil por excelencia-, no sería, desde el sentido común y su lenguaje ordinario, una "inversión"?

63. Es tal vez por lo anterior que, entre otras razones, tribunales arbitrales CIADI han decidido moderar las listas de "inversiones" en los TBIs a través del sacrosanto principio de la buena fe.

\subsection{De la limitación de la lista de "inversiones" de los TBIs a través del sacrosanto principio de la buena fe (¿principio general del derecho internacional?)}

64. No parece aventurado afirmar que el propio sentido común indica que solamente quien obra de buena fe (y no de mala fe) puede ser protegido por las normas que rigen a una sociedad y que conocemos, en su conjunto, como "derecho".

65. One must come to equity with clean hands 29.

66. Ningún inversionista podría invocar las protecciones de un TBI $-\mathrm{y}$, en especial, aquella del trato justo y equitativo de la inversión- si no ha obrado, con relación a su inversión, de buena fe.

67. Este principio ha sido aplicado por tribunales arbitrales CIADI de dos maneras diferentes para limitar la amplitud de la lista de "inversiones" de los TBIs.

29. Ver las reflexiones de la Comisión de derecho internacional sobre este requisito de admisibilidad de las reclamaciones intemacionales: J. Dugard, Sexto informe sobre protección diplomática, Doc. A/CN $4 / 546,11$ de agosto de 2004. Frierdich and Co. Case (Francia c./ Venezuela), Comisión Mixta Francia/Venezuela, laudo del 31 de julio de 1905, Recueil des sentences arbitrales, Vol. 10, p. 54 ; Jarvis (Estados Unidos c./ Venezuela), Comisión Mixta Estados-Unids/Venezuela, laudo de 1905, Recueil des sentences arbitrales, Vol. 9, p. 213. J. Salmon, "Des "Mains Propres" comme condition de recevabilité des réclamations intemacionales", Antuaiure français de droit international, Vol. 10 , 1964, p. 225.

236 Revista Ecuatoriana de Arbitraje 
68. Varios tribunales, en primer lugar, han decidido declararse incompetentes en casos en los cuales los supuestos inversionistas han elaborado montajes societarios con el único objeto de tener acceso a las protecciones del TBI aplicable y al arbitraje CIADI. Los casos TSA Spectrum de Argentina S.A.c./ La República Argentina ${ }^{30}$, Phoenix Action, Ltd. c./ La República Checa y Fraport AG Frankfurt Airport Services Wolrdwide c./ La República de Filipinas ${ }^{31}$ constituyen un buen ejemplo de lo anterior.

69. El tribunal arbitral del caso Phoenix Action, Ltd. c./ La República Checa, en especial, erigió la buena fe-que, según el tribunal, sería un principio general del dexecho internacional- como criterio determinante de la noción de "inversión" de la siguiente manera:

"In the instant case, no question of violation of a national principle of good faith or of international public policy related with corruption or deceitful conduct is at stake. The Tribunal is concerned here with the international principle of good faith as applied to the international arbitration mechanism of ICSID. The Tribunal has to prevent an abuse of the system of international investment protection under the ICSID Convention, in ensuring that only investments that are made in compliance with the international principle of good faith and do not attempt to misuse the system are protected" 32 .

70. Los especialistas en derecho internacional privado no dejarán de entrever la similitud entre la aplicación del principio de la buena fe por los tribunales arbitrales en los casos TSA Spectrum de Argentina S.A.c./ La República Argentina, Phoenix Action, Ltd. c./ La República Checa y Fraport AG Frankfurt Airport Services Wolrdwide c./ La República de Filipinas y el

30. TSA Spectrum de Argentina S.A. c./ la República Argentina, Caso CIADI N ${ }^{\circ}$ ARB/05/5, laudo del 19 de diciembre de 2008, par. 133-162, en línea: <http://ita.law.uvic.ca/documents/TSAAwardSp.pdfs.

31. Fraport AG Frankfurt Airport Services Worldwide c./ La Republica de Filipinas, Caso CIADI ${ }^{\circ}$ ARB/03/25, laudo del 16 de agosto de 2007, par. 383-404, en línea: < http://ita.law.uvic.ca/documents/FraportAward.pdf?.

32. Pheonix, par. 113 
correctivo del "fraude a la ley normalmente competente" 33 . El abuso del sistema de resolución de desavenencias del CIADI equivaldría, en suma, a un fraude a la ley normalmente competente que designa a las cortes nacionales del Estado receptor de la inversión como las únicas con jurisdicción para conocer del fondo de un litigio.

71. En segundo lugar, algunos tribunales arbitrales CIADI han decidido declararse incompetentes ante supuestas inversiones realizadas en violación del derecho del Estado receptor de la inversión. Los casos Inceysa Vallisoletana, S.L. c./ La República de El Salvador 34 , World Duty Free c./ Kenia ${ }^{35}$ y Plama Consortium Limited c./ La República de Bulgaria ${ }^{36}$ ilustran este segundo tipo de limitación a la lista de "inversiones" protegidas según los TBIs.

72. Los dos primeros casos citados más arriba dejan en claro, en especial, que supuestas inversiones realizadas mediante corrupción no pueden ser protegidas. El tribunal arbitral del caso World Duty Free c./ Kenia sostuvo, en particular, lo que sigue:

"In light of domestic laws and international conventions relating to corruption, and in light of the decisions taken in this matter by courts and arbitral tribunals, this Tribunal is convinced that bribery is contrary to the international public policy of most, if not all, States or, to use another formula, to transnational public policy. Thus, claims based on contracts of corruption or on contracts obtained by corruption cannot be upheld by this Arbitral Tribunal"37.

33. P. Mayer y V. Hcuzé, Droit international privé, Montchrestien (2007), pp. 189-195.

34. Inceysa Vallisoletana, S.L. c./ La República de El Salvador, Caso CIADI ${ }^{\circ}$ ARB/03/26, laudo del 2 de agosto de 2006, par. 208-337, en linea: _http://ita.law.uvic.ca/documcnts/Inceysa_Vallisoletana pdf?.

35. World Duty Free Company Limited c./Kenya, Caso CIADI No. ARB/00/7, laudo del 4 de octubre de 2006, en lined: <http:/ita.law.uvic.ca/documents/WDFv.KenyaAward.pdf $>$ (en adelante "World Duty Free").

36. Plama Consortium Limited c./ La República de Bulgaria, Caso CIADI N ${ }^{\circ}$ ARB/03/24, laudo del 27 de agosto de 2008, par. 194-305, en linea:

<http://ita.law.uvic.ca/documents/PlamaBulgariaAward.pdfs.

37. World Duty Free, par. 157. 
73. Parece ser que el sacrosanto y vago principio de la buena fe se traduce, en los casos citados, en la condena del abuso del derecho y la corrupción, y el respeto debido a las leyes y los reglamentos de los Estados receptores de la inversión.

74. Dichos casos nos recuerdan la decisión del árbitro Lagergren en el famoso caso $\mathrm{CCI} \mathrm{N}^{\circ} 1110$. En dicho caso, Lagergren, ante una clara situación de corrupción, decidió declararse incompetente para conocer del fondo del asunto en los siguientes términos:

"After weighing all the evidence I am convinced that a case such as this, involving such gross violations of good morals and international public policy, can have no countenance in any court either in the Argentine or in France, or, for that matter, in any other civilised country, nor in any arbitral tribunal. Thus, jurisdiction must be declined in this case. It follows from the foregoing, that in concluding that I have no jurisdiction, guidance has been sought from general principles denying arbitrators to entertain disputes of this nature rather than from any national rules on arbitrability. Parties who ally themselves in an enterprise of the present nature must realise that they have forfeited any right to ask for assistance of the machinery of justice (national courts or arbitral tribunals) in settling their disputes" 38 .

75. La decisión de Lagergren fue criticada y, según se enseña, superada. Se dijo que Lagergren, en lugar de declararse incompetente, ha debido decidir el fondo y anular el contrato en cuestión por objeto ilícito.

76. ¿Los tribunales CIADI que han aplicado el principio de la buena fe han debido declararse competentes y luego rechazar las pretensiones del inversionista en razón de su mala fe? ¿La mala fe del inversionista, en otras palabras, plantea un problema de jurisdicción o de fondo?

38. Laudo de 1963 en el Caso CCI No. 1110, Arbitration International, Vol. 10 No. 3 (1994), p. 294, par. 24. 
77. Los especialistas en "derecho internacional de las inversiones" quizás sugerirían que la respuesta dependerá de cómo estén escritos los TBIs aplicables (subjetivismo). Lo que, como quiera que esto sea, es cierto es que un tribunal arbitral en materia de inversión no puede anular el TBI y que es más económico para las partes (por razones obvias) que la mala fe del inversionista sea tratada como un tema de jurisdicción que como un problema de fondo.

78. Nosotros queremos creer, en voz alta y borrador, como lo afirma el tribunal arbitral del caso Phoenix Action, Ltd.c./ La República Checa, que existe un principio general de derecho internacional según el cual un inversionista de mala fe no puede ser protegido.

79. Los comentarios anteriores ponen de manifiesto que los problemas fundamentales del naciente derecho internacional de las inversiones se hallan en la incertidumbre en cuanto a sus fuentes y al método de interpretación de los TBIs.

80. Abogados y árbitros, de un lado, se sienten incómodos al fundar argumentos y decisiones sobre el sentido común, los principios generales 0 , en general, todo elemento extrínseco a los TBIs y otros tratados (como el Convenio de Washington) aplicables. El positivismo imperante en la materia es apabullante. Abogados y árbitros, en suma, parecerían temer la censura y el ridículo al no fundamentar y motivar sus posiciones sobre la base de normas jurídicas escritas (tratados, leyes o precedentes).

81. ¿El naciente derecho internacional de las inversiones es alérgico al sentido común y a los principios generales? 
82. ¿No hemos olvidado, a fin de cuentas, lo prescrito en el artículo $38^{39}$ del Estatuto de la Corte Internacional de Justicia?

83. Abogados y árbitros, de otro lado, se sienten obligados, en materia de inversión, a seguir los métodos de interpretación de los tratados prescritos en los artículos $31^{40}$ y $32^{41}$ de la Convención de Viena sobre el Derecho de los Tratados. ¿Estas normas, sin embargo, describen los únicos métodos disponibles ( $y$ el orden de su aplicación) para interpretar los TBIs y por ende definir, entre muchas otras, la noción de "inversión"? ¿Una definición de "inversión" resultante de una interpretación de un TBI de conformidad con dichas normas que sea contraria a aquella del sentido común y el lenguaje ordinario no sería irrazonable?

39. "I. La Conte, cuya función es decidir conforme al derecho internacional las controversias que le sean sometidas, deberá aplicar:

a. las convenciones internacionales, sean generales o particulares, que establecen reglas expresamente reconocidas por los Estados litigantes;

b. La costumbre internacional como prueba de una práctica generalmente aceptada como derecho:

c. los principios generales de derecho reconocidos por las naciones civilizadas;

d. Las decisiones judiciales y las doctrinas de los publicistas de mayor competencia de las distintas naciones, como medio auxiliar para la determinación de las reglas de derecho, sin perjuicio de lo dispuesto en el Articulo 59.

2. La presente disposición no restringe la facultad de la Corte para decidir un litigio ex aequo et bono, si las partes asi lo convinieren".

40. "Regla general de interpretación.

1. Un tratado deberá interpretarse de buena fe conforme al sentido corriente que haya de atribuirse a los términos del tratado en el contexto de éstos y teniendo en cuenta su objeto y fin.

2. Para los efectos de la interpretación de un tratado, el contexto comprenderá, además del texto, incluidos su preámbulo y anexos:

a) todo acuerdo que se refiera al tratado y haya sido concertado entre todas las partes con motivo de la celebración del tratado;

b) todo instrumento formulado por una o más partes con motivo de la celebración del tratado y aceptado por las demás como instrumento referente al iratado.

3. Juntamente con el contexto, habrá de tenerse en cuenta:

a) todo acuerdo silerior entre las partes acerca de la interpretación del tratado o de la aplicación de sus disposiciones;

b) toda práctica ulteriomente seguida en la aplicación del tratado por la cual conste el acterdo de las partes acerca de la interpretación del tratado;

c) toda forma pertinente de derecho internacional aplicable en las relaciones entre las partes.

4. Se dará a un término un sentido especial si consta que tal fue la intención de las partes".

4t. "Medios de interpretación complementarios. Se podrá acudir a medios de interpretación complementarios, en particular a los trabajos preparatorios del tratado y a las circunstancias de su celebración, para confirmar el sentido resultante de la aplicación del artículo 31, o para determinar el sentido cuando la interpretación dada de conformidad con el articulo 31 :

a) deje ambiguo u oscuro el sentido;

b) conduzca a un resultado manifiestamente absurdo o irrazonable". 
84. Una opinión disidente reciente ${ }^{42}$ da luces interesantes sobre los principios que deben ser seguidos en materia de interpretación de tratados:

"Incidental divergences with fellow arbitrators do not, in my view, necessarily require written expression. I have never before felt impelled to dissent. In this instance, I unfortunately find myself in disagreement with respect to the decisive proposition advanced by my two esteemed colleagues, which as far as I can see could be obtained only by an impermissible rewriting of the Treaty we are bound to apply. [...]

HEP, a Croatian Government-owned joint stock company, has brought this arbitration against Slovenia under an agreement signed by Ministers representing Slovenia and Croatia on 19 December 2001. HEP properly refers to this instrument as a treaty, and indeed invokes the Vienna Convention on the Law of Treaties ("the VCLT") as applicable to its interpretation. It therefore seems appropriate to refer to it as "the Treaty." HEP claims damages on account of non-delivery of electricity as from 1 July 2002 to 19 April 2003. And yet:

(i) HEP is not a signatory ofthe Treaty;

(ii) the Government of Slovenia does not produce or market electricity; and

(iii) above all, the Treaty simply does not contain an undertaking to deliver electricity as from 1 July 2002.

The first two problems would perhaps not have sufficed to cause me to part ways with the majority; with some legal footwork they could have been sidestepped. (Whether the majority have indeed succeeded in finding a solid path remains, however, a matter of doubt; see Paragraph 75 below.) But to conclude that a treaty which does not establish an obligation of delivery nevertheless creates liability for non-delivery is one long bridge too far. I find no words in the Treaty that support such an extraordinary outcome.

I have naturally sought an overriding cause for this sharp divergence. It appears to me that there are in fact two related causes.

42. Opinion individual de Jan Paulsson en el Caso CIADI N ${ }^{\circ}$ ARB/05/24 entre Hrvatska Elektroprivreda D.D. c./ la República de Eslovenia, decisión sobre la interpretación del tratado del 12 de junio de 2009, en linea: <http://ta,law.uvic.ca/documents/Hrvatska-Interpretation-Paulsson.pdf $>$ 
The first is a basic difference of approach, in which the majorit$y^{\prime}$ s natural desire to reach a result that they consider fair and reasonable leads them to imply terms that are not in the Treaty, to ignore terms that are in the Treaty, and to give retroactive effect to a Treaty when neither its express terms nor its object require retroactivity. I would have great faith in my colleagues, whom I know well and whose views I often share, if they were entrusted with a mission of determining matters of fairness and reasonableness. Yet this Tribunal has not been authorised to decide the dispute ex aequo bono (as would have been required under Article 42(3) of the ICSID Convention). Even if the contrary were true, we would not be in a position to exercise that discretion at this stage of the case. The transformations attendant on the breakup of Yugoslavia gave rise to complex disputes that, as of the signing of the Treaty, had been ongoing for a decade. Originally, nuclear plants had been envisaged to be built in Croatia as well as in Slovenia. The plan for a Croatian plant was abandoned; Croatia became a customer of the plant in Slovenia, but no longer as a fellow federated State within Yugoslavia. This led to predictable divergences. Slovenia invoked international obligations requiring significant investments for Krsko NPP ("the Plant"). (This factor is conspicuously absent from the majority's account under the rubric "The Nature of the Dispute", paras. 6-15.) HEP objected that the expenses had been budgeted unilaterally, and were therefore not opposable to it. On the Slovenian side, it was said that HEP's unjustified interruption of off-take threatened the Plant's financial ruin. HEP retorted that the cause for these difficulties was a failure to respect its right of participation in matters of governance. The list goes on, and the complications are endless. I simply do not see how we could at this stage assess the equities of a long narrative where sharply opposed theses have not been fully presented or examined.

The second cause of my disagreement is a difference in our understanding of the bargain set out in Exhibit 3 to the Treaty. For the majority, that bargain includes a "critical date" of 30 June 2002. If electricity did not begin to flow to HEP on that date, they apparently believe, it became inequitable to enforce the other terms of the bargain which, in their view, were all to take effect as of that

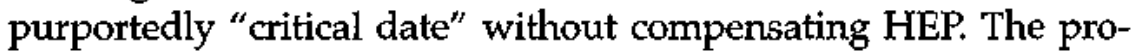
blem with this analysis is not only that it is incorrect in principle 
to rewrite the Treaty to comport with post hoc notions of equity, but that it is unnecessary to do so. The Treaty does not say that the other elements of Exhibit 3 take effect on 30 June 2002. The perceived unfairness to HEP simply does not arise.[...] The majority apparently believe that it would be inequitable for HEP not to receive electricity on the same date that it assumed $50 \%$ responsibility for modernisation costs and exchanged mutual waivers of claims with NEK. But there is no need to imply terms or to introduce retroactivity to achieve that result: the Treaty's text already provides for all of those elements to take effect on the same date. The fact that that date is the Treaty's entry into force, rather than 30 June 2002, does not change the essence of the Exhibit 3 bargain; nor does it render it less equitable. On a proper reading of the Treaty, HEP starts sharing responsibility for costs as of the day electricity deliveries commence, after the Treaty's entry into force.

I moreover feel constrained to express my disappointment upon reading paras. 11-12 of the majority's Decision, which appears in what one would expect to be an objective introductory section of the Decision ("The Nature of the Dispute"). These passages are, in my view, anything but neutral; they read like pleadings on behalf of HEP and seem intended to induce the reader into a strong intuitive sense of the essential faimess of HEP's position, and thus to lay the groundwork for a section of the Decision, commencing with para. 191, entitled "Good Faith". The fact is that these two paragraphs express views of great controversy. Naturally arbitrators have the authority to resolve controversies, but only after examining both sides of the debate and giving reasons for their findings. Here the majority seem to reverse-engineer from their desired outcome, which becomes clear when one reaches para. 191 and its remarkable statement that in treaty interpretation good faith is "the core principle about which all else resolves". This follows the even more curious affirmation in para. 176 that the degree of clarity of a treaty term does not give it "greater or lesser force" ${ }^{\prime \prime}$ I shall revert to this matter in due course (see Paragraphs 23 and $39-51$ below) $)^{\prime \prime}$.

85. ¿No podría llegarse a una definición de "inversión" cercana a la que provee el sentido común interpretando la lista de "inversiones" de un TBI de conformidad con la finalidad y el contexto de dicho tratado?

244 REvista ECUATORIANA DE ARBTTRAJE 
Por un regreso al sentido ordinario de la palabra "inversión"

86. Nosotros, como quiera que sea, estamos convencidos, dada la manera en que funcionan las comunidades humanas, que la justicia se halla más cerca del sentido común y del lenguaje ordinario (jusnaturalismo moderado) que de las tecnicidades, muchas veces confusas y artificiales, del lenguaje jurídico. 
